\title{
Natura fisica della scossa all' ipocentro di alcuni terremoti profondi nel Mediterraneo
}

\author{
D. Di Filippo - F. Peroxide
}

ricevito il lo dicembre 1959

Il Mediterraneo si riteneva privo di centri sismici profondi. Clteriormente, però, ne sono stati posti in evidenza un rerto numero di cui alcuni hamno dato luogo a terremoti di notevole intensità; questo fatto ha originato una serie di ricerche da parte di studiosi di geofisica; ricordiamo tra gli altri Gutenberg, Richter, Caloi, Peterschmitt. Le conchusioni a cui si è giunti hanno consentito di stabilire che mentre la dorsale appenninica e l'Adriatico presentano terremoti caratterizzati da un mecomismo

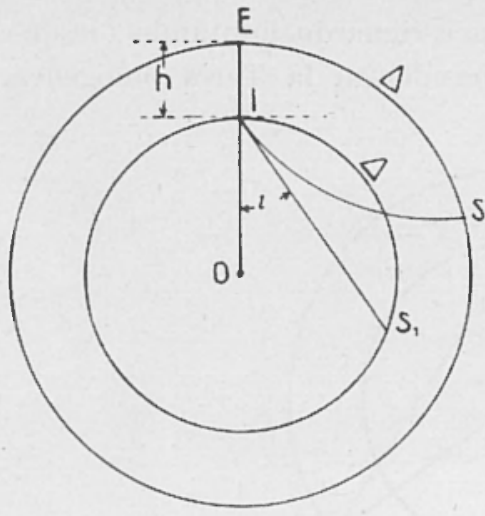

Fig. 1

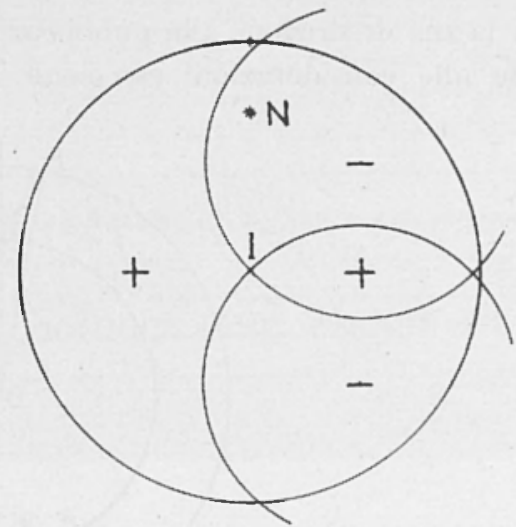

Fig. 2

di sollevamento e di sprofondamento rispettivamente, i terremoti profondi che si sono verificati nel Basso Tirreno e nel Mediterraneo occidentale presentano in prevalenza lo schema della frattura. Partendo da queste considerazioni ci siamo proposti di approfondire la questione indagando sul meccanismo whe ha dato origine alla scossa per il maggior 
numero di terremoti possibili onde porre in evidenza la giacitura e l'orientazione di eventuali faglie a grande profondità, interessanti la zona che separa l'Europa dal continente africano.

Il procedimento seguito è doruto ad uno di noi; esso consiste nella estensione ai terremoti profondi del metodo proposto da Byerly per i

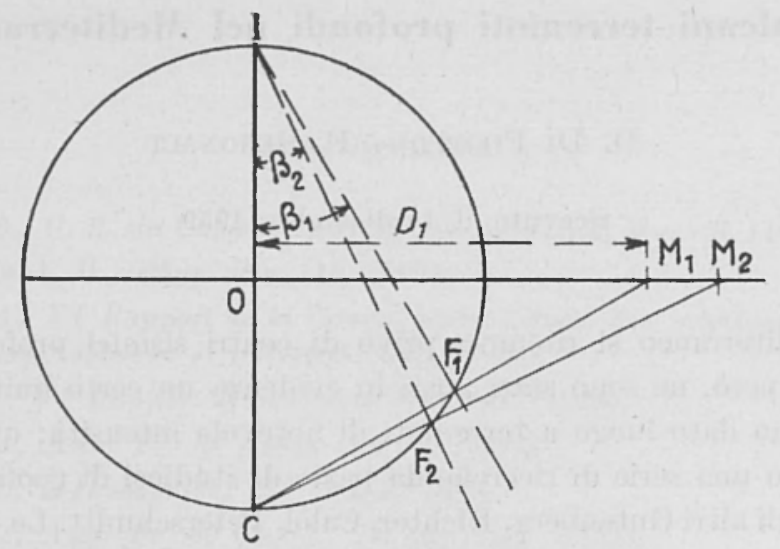

Fig. 3

terremoti superficiali; trattandosi di un metodo ormai noto rimandiamo per la sua descrizione alla pubblicazione a riguardo, limitandoci in questa sede alle considerazioni essenziali. Considerata la Terra omogenea, e

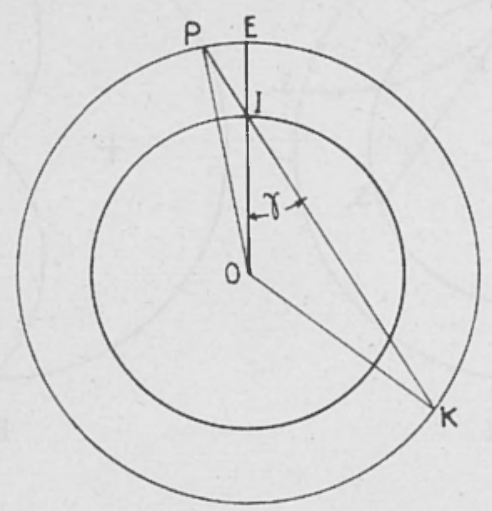

Fig. 4

quindi i raggi sismici rettilinei, se rappresentiamo con i due cerchi concentrici di fig. 1 le sezioni della Terra e della sfera ipocentrale si deve sostituire al raggio sismico $I S$ il corrispondente rettificato $I S_{1}$. 
$S_{1}$ rappresenterà sulla sfera ipocentrale la posizione della stazione $S$, mentre la distanza $\Lambda_{i}$ si calcolerà, nota la distanza epicentrale $\Delta$, le

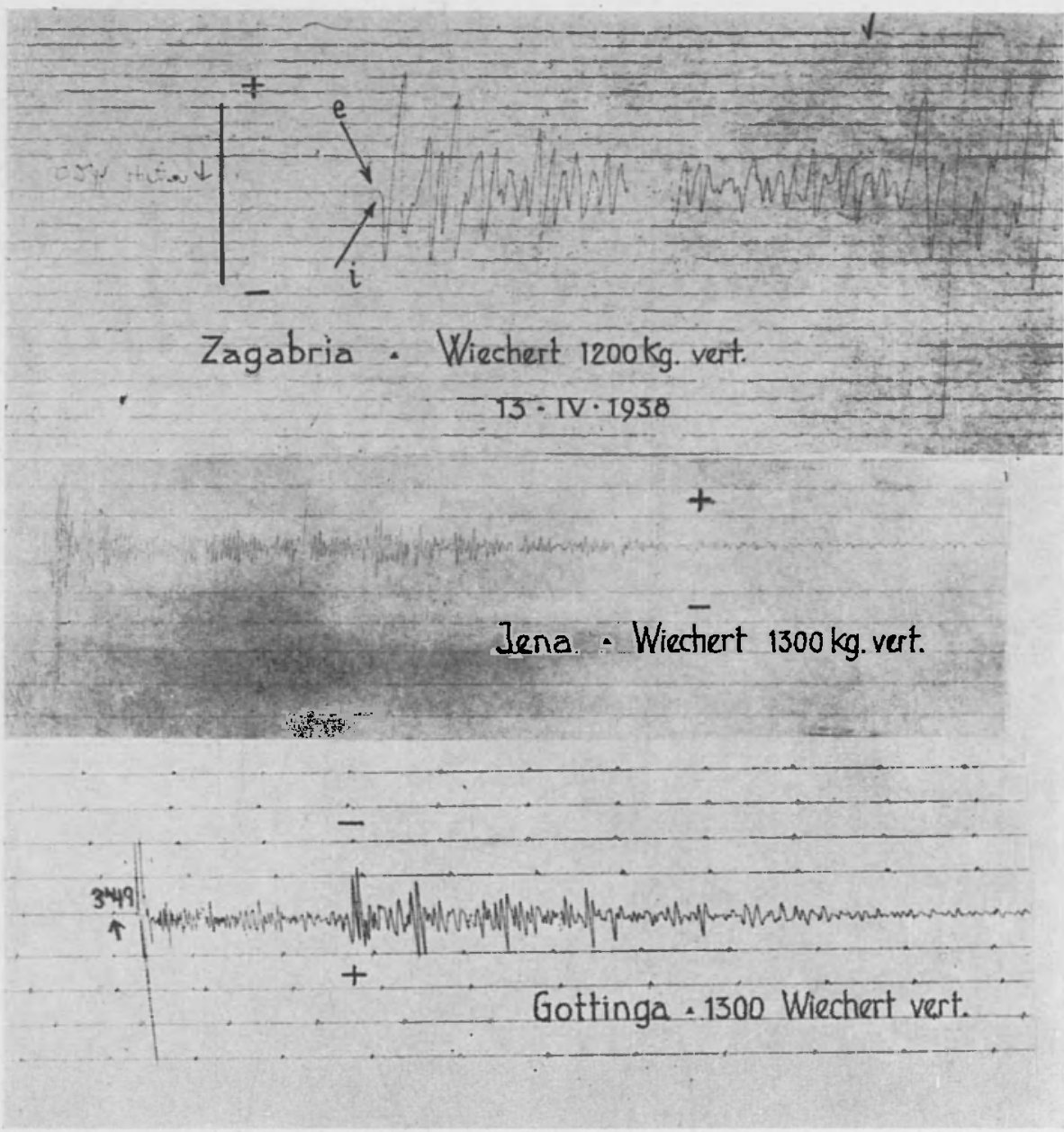

Fig. 5

coordinate ipocentrali e la velocità $V_{h}$ allipocentro, integrando la relazione fondamentale che regola il propagarsi dei raggi sismici:

$$
\frac{r_{h} \operatorname{sen}}{V_{h}} i_{h}=r_{0} \frac{\operatorname{sen} i_{0}}{V_{0}}
$$


Infatti ricordando la relazione di Bemndorf sen $i_{o}=\frac{r_{o}}{v_{n}}$, ove $v_{n}$ e la velocità apparente alla distanza $\Delta$, si ottiene

$$
\operatorname{sen} i_{h}=\frac{r_{0} V_{h}}{r_{h} \frac{d \Delta}{d t}}
$$

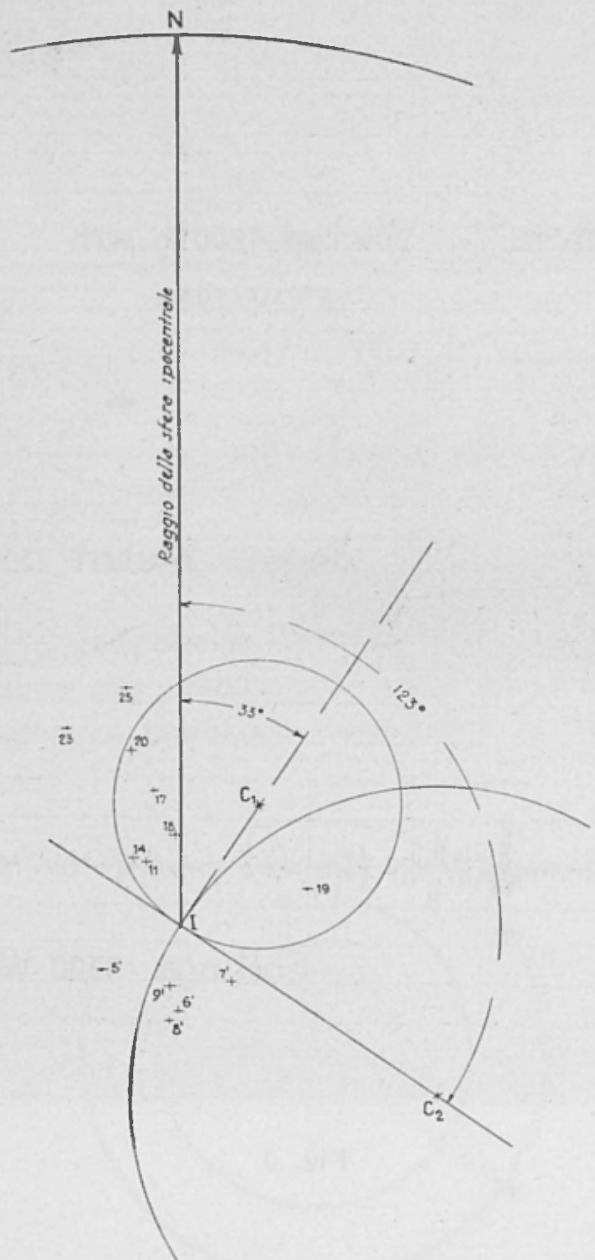

Fig. 6

che permette di calcolare l'angolo $i_{h}$ all'ipocentro del raggio sismico considerato; l'angolo complementare di $i_{h l}$ fornirà direttamente in gradi la distanza $A_{1}$ che in proiezione stereografica sul piano equatoriale della 
sfera ipocentrale, risulterà data dal valore di cot $i_{h t}$. Per quanto riguarda l'azimut di $S_{1}$ rispetto al meridiano passante per l'ipocentro e diretto verso il Nord, esso coincide con quello calcolato per la stazione $\mathcal{S}$ che si considern.

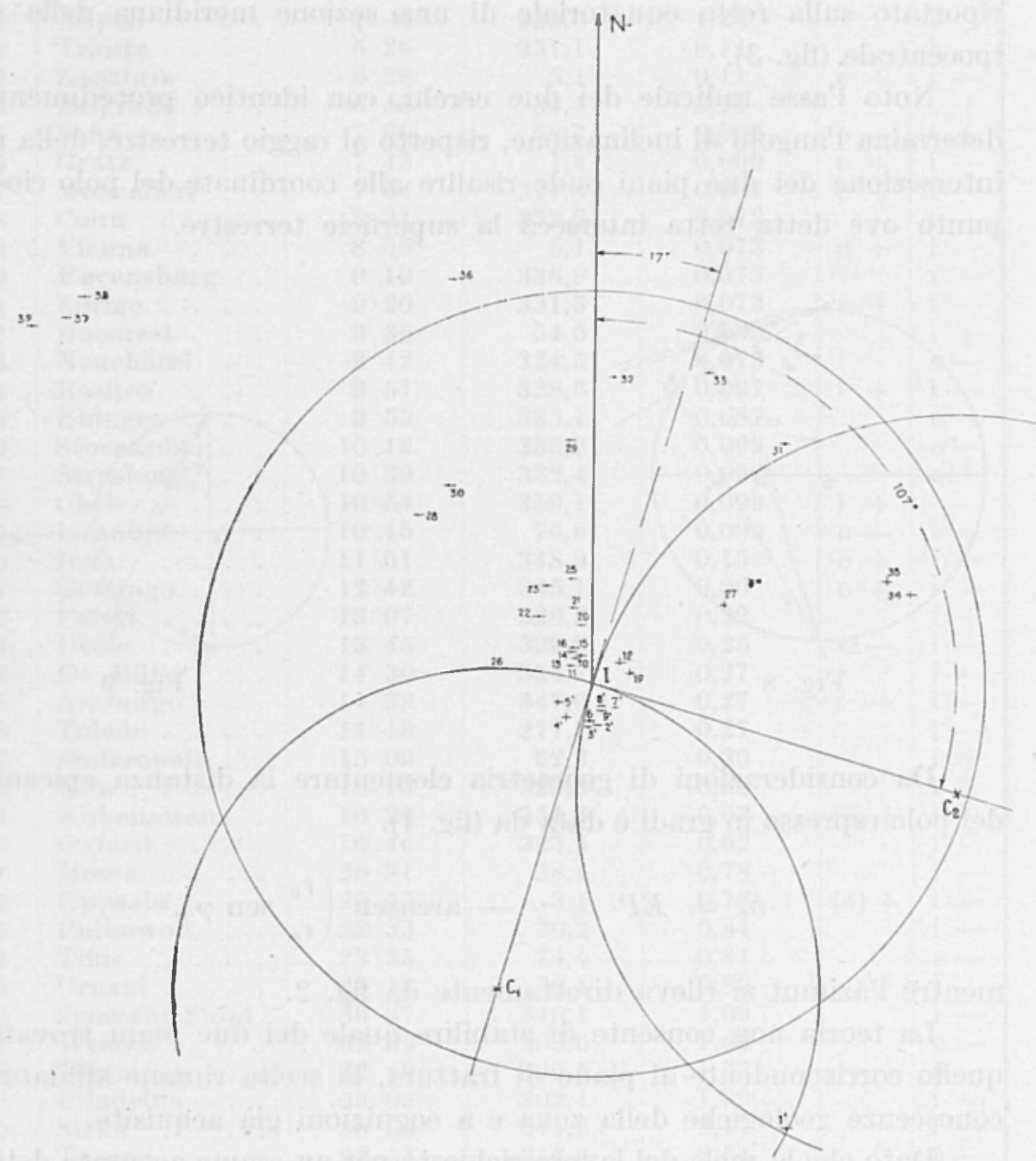

Fig. 7

Ottenuti gli elementi necessari per riportare le posizioni delle stazioni in proiezione stereografica con l'ipocentro centro della figura, si riporta per ogni stazione il segno del primo impulso, e se la natura del 
meccanismo della scossa è unir frattura, la distribuzione degli impulsi iniziali consente di individuare la posizione dei due cerchi, ortogonali tra loro e passanti per l'ipocentro, che delimitano le quattro zone di dilatazione e compressione tali che a zone opposte corrispondano impulsi uguali (fig. 2). Gli angoli che le tangenti ai cerchi per il centro di figura formano con la direzione del Nord forniscono l'orientamento dei piani, mentre l'inclinazione di essi rispetto al raggio terrestre per l'ipocentro si determina a partire dalla conoscenza del diametro dei cerchi, proiezione riportato sulla retta equatoriale di una sezione meridiana della sfera ipocentrale (fig. 3).

Noto l'asse radicale dei due cerchi, con identico procedimento si determina l'angolo di inclinazione, rispetto al raggio terrestre, della retta intersezione dei due piani onde risalire alle coordinate del polo ciò̀ del punto ove detta retta interseca la superficie terrestre.

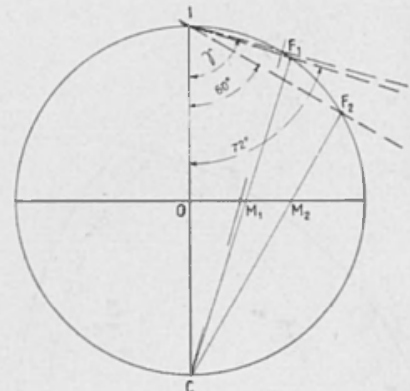

Fig. 8

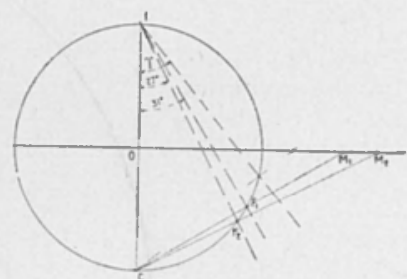

Fig. 9

Da considerazioni di geometria elementare la distanza epicentrale del polo espressa in gradi è data da (fig. 4).

$$
\delta_{p}=E P=\gamma-\operatorname{arcosen}\left(\frac{r_{h}}{r_{o}} \operatorname{sen} \gamma\right)
$$

mentre l'azimut si rileva direttamente da fig. 2.

La teoria non consente di stabilire quale dei due piani trovati sia quello corrispondente al piano di frattura, la scelta rimane affidata alle conoscenze geologiche della zona e a cognizioni già acquisite.

Dato che la mole del lavoro richiesto per un esame accurato del problema è notevole, in questo primo lavoro ci siamo limitati ad esaminare quattro dei più forti terremoti profondi e precisimente quello delle isole Lipari del 13.IV.1938, del Basso Tirreno del 14.III.1941, del Golfo di Policastro del 26.XIT .1952 e della Spagna meridionale del 29.III .1954. 
Tabella I

\begin{tabular}{|c|c|c|c|c|c|c|}
\hline N. & Stazione & Io & $a^{0}$ & $\begin{array}{c}\operatorname{Cotg} i_{h} \\
o \\
\operatorname{tang} 1 / A_{1}\end{array}$ & $\begin{array}{c}\text { Primo } \\
\text { im- } \\
\text { pulso }\end{array}$ & $\begin{array}{c}\text { Se- } \\
\text { condo } \\
\text { im- } \\
\text { pulso }\end{array}$ \\
\hline 1 & Napoli & $10+1^{\prime}$ & 335,6 & 1.24 & & $e-$ \\
\hline 2 & Trieste & 626 & 351,1 & 0,11 & & $i$ \\
\hline 3 & Zagabria. . & 629 & 5,1 & 0,11 & e + & $\mathrm{i}-$ \\
\hline 4 & Belgrarlo. . . & 643 & 34,1 & 0,11 & & $\mathrm{i}+$ \\
\hline 5 & Sofia & 700 & 58,7 & 0,009 & $\mathbf{e}-$ & $\hat{\mathbf{i}}+$ \\
\hline 6 & Gratz & 743 & 1,4 & 0,099 & e + & $\mathrm{i}-$ \\
\hline 7 & IIonealieri & 754 & 317,8 & 0,087 & e + & $\mathrm{i}-$ \\
\hline 8 & Coira . & 831 & 332,9 & 0,073 & & $\mathrm{i}-$ \\
\hline 9 & Vienna & 855 & 5,1 & 0,073 & e + & $\mathrm{i}-$ \\
\hline 10 & Revenshurg . & $9 \quad 19$ & 336,9 & 0,073 & & $\mathrm{i}-$ \\
\hline 11 & Zurigo.- . & 920 & 331,3 & 0,073 & e + & $\mathrm{i}-$ \\
\hline 12 & Bucarest & 936 & 54,5 & 0,073 & & $\mathrm{i}+$ \\
\hline 13 & Neuchâtel . & 942 & 324,5 & 0,073 & & e - \\
\hline 14 & Basilea . . & 951 & 328,5 & 0,087 & $\mathrm{i}+$ & $\mathrm{i}-$ \\
\hline 15 & Ebingen & 953 & 335,1 & 0,087 & & $e-$ \\
\hline 16 & Stoccarda . & $10 \quad 19$ & 336,2 & 0,099 & & $B-$ \\
\hline 17 & Strasburg. : & 1039 & 332,4 & 0,099 & & $\theta+$ \\
\hline 18 & ('heh) . . & 1054 & $359, \mathrm{l}$ & 0,099 & $\mathrm{i}+$ & \\
\hline 19 & Istanbul . . . & 1045 & 76,6 & 0,099 & e - & $\mathrm{i}+$ \\
\hline 20 & Iena. & 1151 & 348,9 & 0,15 & e + & i \\
\hline 21 & Gottinga . & 1242 & 345,1 & 0,20 & e + & $\mathrm{i}-$ \\
\hline 22 & Parigi. . . & 1307 & 320,3 & 0,22 & & $\mathrm{i}-$ \\
\hline 23 & Uecle . . & 1345 & 329,9 & 0,25 & ei- & $\mathrm{i} \cdot-$ \\
\hline 24 & De Bilt . . & 1430 & 334,7 & 0,27 & & $\mathrm{i}-$ \\
\hline 25 & Amburgo & $1+38$ & 347,6 & 0,27 & i - & i - - \\
\hline 26 & Toledo. & $14+9$ & 277,1 & 0,27 & & $\mathrm{i}-$ \\
\hline 27 & Sinferopoli . . & 1506 & 62,3 & 0,39 & & $\mathrm{i}+$ \\
\hline 28 & kew. . & $16 \quad 13$ & 323,3 & 0,62 & & $\mathrm{i}-$ \\
\hline 29 & Kohenawen & 1626 & 354,6 & 0,62 & (e) + & $\mathrm{i}-$ \\
\hline 30 & Oxford . & 1646 & 323,5 & 0,62 & & $\mathrm{i}-$ \\
\hline 31 & Mosca . & 2031 & 38,4 & 0,78 & & $\mathrm{i}-$ \\
\hline 32 & Ippsiala . & 2045 & 3,1 & 0,78 & (e) + & $i=$ \\
\hline 33 & l'ulkowo . & $22 \quad 33$ & 20,2 & 0,84 & & $\mathrm{i}-$ \\
\hline 34 & Tiflis & 2335 & 74,4 & 0,84 & & e- \\
\hline 35 & Grozni & 2313 & 70,4 & 0,89 & & $\mathrm{i}+$ \\
\hline 36 & Scoreshy Sund. & 3637 & 340,1 & 1,09 & & $\mathrm{i}-$ \\
\hline 37 & Weston & $62 \quad 39$ & 303,6 & 1,63 & & $\mathrm{e}--$ \\
\hline 38 & Wermont : & 6249 & 306,5 & 1,63 & & e - \\
\hline 39 & Filadelfia & 6505 & 302,1 & 1,69 & & $\mathrm{i}-$ \\
\hline 40 & Sitka & 8055 & $3+4,2$ & 2,17 & & $\mathrm{i}-$ \\
\hline 41 & Tueson & 9254 & 316,6 & 2,54 & & e - \\
\hline 42 & M. Wilson . & 9457 & 322,7 & 2,58 & & i - - \\
\hline 43 & Pasadena : & 9504 & 322,8 & 2,58 & & $\mathrm{i}-$ \\
\hline
\end{tabular}


TERREMO'TO DELAE ISOLE LIPARI DEI, 13.IV .1938.

Il terremoto è stalto oggetto di studio da parte di molti ricercatori che concordemente hamno fissato la profondità ipocentrale ad un valore superiore ai $230 \mathrm{~km}$.

Tabella 2

\begin{tabular}{|c|c|c|c|c|c|c|}
\hline N. & Osservatori & & $\begin{array}{c}\text { Imp. } \\
\text { ini- } \\
\text { ziali }\end{array}$ & 10 & $\alpha^{\circ}$ & 1.0 \\
\hline 1 & Tunisi & . & - & 20,277 & 2220,93 & - \\
\hline 2 & Napoli . . . & .. & - & 2.910 & 33,55 & -- \\
\hline 3 & Polistena . & . . & + & 2.105 & 86.26 & - \\
\hline 4 & Roma... & . . & - & 3,478 & 4,41 & - \\
\hline 5 & Prato & . & - & 5,500 & 352,38 & -- \\
\hline 6 & Padova. & 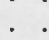 & - & (6),964 & 358,51 & to, 60 \\
\hline 7 & Marsiglia. . & . & + & 7,025 & 315,66 & 5,30 \\
\hline 8 & 'Treviso & . . & - & 7,228 & 0,33 & 7,30 \\
\hline 9 & Trieste & . & - & 7.311 & 8,98 & 8,10 \\
\hline 10 & Moncalieri & . . & + & 7,342 & 334,71 & 8,40 \\
\hline 11 & Coira . . & . . & - & 8,623 & 346,55 & 17,48 \\
\hline 12 & Kurigo . & .. & - & 9,298 & 344,98 & 22,00 \\
\hline 13 & Neuchâtel & . & + & 9,357 & 337,80 & 22,40 \\
\hline 14 & Basilea . . & . . & - & 9,682 & 341,47 & 24,52 \\
\hline 15 & Monaco & . & - & 9,715 & 357,96 & 24,72 \\
\hline 16 & Clermont & . & - & 9,986 & 320,20 & 26,60 \\
\hline 17 & Vienna & . & - & 10.278 & $16,0]$ & 28,20 \\
\hline 18 & Budapest & . . & - & 10,365 & 26,76 & 29,12 \\
\hline 19 & Bucarest & 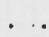 & + & 12.038 & 55.79 & 37.70 \\
\hline 20 & Jena & . & - & 12,504 & 358,43 & 40,00 \\
\hline 21 & Toledo. & . & + & 12,584 & $281 \quad .37$ & 40.40 \\
\hline 22 & Gottinga & . & . & 13,195 & 351,37 & 43,46 \\
\hline 23 & Eccle & 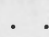 & 一 & 13,522 & 338,58 & 45,10 \\
\hline 24 & De Bilt & r. & - & $14,49]$ & 342,74 & 49,96 \\
\hline 25 & Copenaghen . & . . & . & 17,245 & 0,62 & 63,00 \\
\hline
\end{tabular}

I nostri calcoli sono stati condotti a partire dai seguenti valori ricavati da Caloi-Giorgi ottenuti come media ponder"ale dei risultati relativi a due diversi metodi:

$$
\begin{aligned}
& \varphi_{o}=39021^{\prime}, 6 \pm 2^{\prime}, 6 \mathrm{~N} \text { (latit. geografica) } \\
& \lambda_{o}=15^{0} 10^{\prime}, 3 \pm 1^{\prime}, 6 \mathrm{E} \\
& H_{o}=02^{\mathrm{h}} 45^{\mathrm{tm}} 50^{\mathrm{s}}, 1 \pm 1^{\mathrm{s}}, 3 \\
& h=290,9 \pm 7,8 \mathrm{~km} \text {. }
\end{aligned}
$$


L'esame dei sismogrammi ha posto in evidenza che il forte impeto presente in tutte le stazioni (anche le più lontane), ̀̀ preceduto in alcuni osservatori più vicini o meglio attrezati, da una leggera emersio spesso di verso opposto all'impeto (vedi fig. 5). $\Lambda$ bbiamo creduto opportuno tener conto anche di tale lieve emersio per le stazioni che ne consentivano il rilievo, di conseguenza sono state eseguite due distinte determinazioni che hanno entrambe condotto ad uno schema di frattura.

I dati necessari alle rappresentazioni cartografiche della distribuzione degli impulsi iniziali sono riportati nella tabella 1 ove per le velocità apparenti ci siamo serviti delle dromocrone di Jeffreys, mentre la velocità all'ipocentro dell'onda $P$ è stata fissata al valore di $8,50 \mathrm{~km} / \mathrm{sec}$ consigliato da Gutenberg.

Tabella 3

\begin{tabular}{|c|c|c|c|c|c|c|c|c|c|}
\hline$N$. & Stazi & ione & & & & fo & $\alpha^{0}$ & $\begin{array}{c}\text { Cotang } i_{h} \\
o \\
\operatorname{tang} \frac{1}{2} \cdot 1_{1}\end{array}$ & $\begin{array}{l}\text { Im- } \\
\text { pulso } \\
\text { ini- } \\
\text { ziale }\end{array}$ \\
\hline I & Messina & . & . & . & . & 10,76 & $180^{\circ}, 0$ & 0,978 & $\mathrm{i}-$ \\
\hline 2 & Roina. & . & 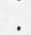 & . & & 3,01 & 310,5 & 0.669 & $\mathrm{i}-$ \\
\hline 3 & Firenze. & . & 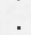 & 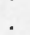 & 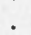 & 4,98 & 320,6 & $0,2+0$ & $\mathrm{i}-$ \\
\hline 4 & Jrato. & . & 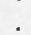 & . & 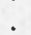 & 5,13 & 321.1 & 0,230 & $e+$ \\
\hline 5 & Bologna & . & . & 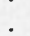 & . & 5,50 & 326,7 & 0,225 & i + \\
\hline 6 & Trieste & - & & . & " & 5,82 & 347,6 & 0,215 & e \\
\hline 7 & Belgrado &. & & . & . & 6,06 & 33,7 & 0,215 & $i$ \\
\hline 8 & Atene. & . & 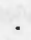 & . & . & 6.67 & 75,2 & 0,215 & $\mathrm{i}-$ \\
\hline 9 & \%urigo & .. & 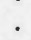 & . & . & 8,96 & 329.0 & 0,215 & $\mathrm{i}-$ \\
\hline 10 & Neuchâtel & . & . & . & . & 9,40 & 321,2 & 0,215 & e - \\
\hline II & Stocearda & .. & 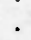 & 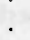 & . & 9,91 & 334,8 & 0,215 & ei - \\
\hline 12 & Besancon. & . & . & . & . & 10,05 & 319,6 & 0,215 & et \\
\hline 13 & Strasburgo & & 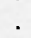 & 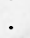 & . & 10,26 & 329,7 & 0,215 & i -- \\
\hline 14 & Algeri . & & 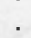 & . & . & 10,32 & 256,2 & 0,220 & ei $t$ \\
\hline 15 & Elwan & & & 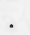 & . & 16,39 & 123,1 & 0,669 & $\mathrm{i}-1$ \\
\hline 16 & Tamarrasett & & . & . & . & 19.11 & 209.4 & 0,812 & ei \\
\hline 17 & Wittewell & . & 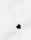 & 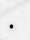 & . & 14,27 & 337,2 & 0,360 & e \\
\hline 18 & Kiruna . & 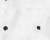 & 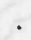 & & . & 28,07 & 3.9 & 1,049 & $\mathrm{i}$ \\
\hline
\end{tabular}

La fig. 6 mostra la distribuzione dei primi impulsi riferiti alle emersio, mentre la fig. $\tau$ ̀̀ il risultato dell'esame degli impeti. Le figure 8 e 9 damno gli angoli di inclinazione nei due casi. In definitiva $i$ valori ottenuti sono:

$$
\begin{array}{ll}
\alpha_{1}{ }^{\prime}=33^{\circ} \mathrm{E} & \beta_{1}{ }^{\prime}=60^{\circ} \\
\alpha_{0}{ }^{\prime}=123^{\circ} \mathrm{E} & \beta_{2}{ }^{\prime}=720 \\
\alpha_{1}{ }^{\prime \prime}=17^{\circ} \mathrm{E} & \beta_{1}{ }^{\prime \prime}=27^{\circ} \\
\alpha_{2}{ }^{\prime \prime}=107^{\circ} \mathrm{E} & \beta_{2}{ }^{\prime \prime}=31^{\circ}
\end{array}
$$

ove gli apici si riferiscono alla $1^{\mathrm{a}}$ e alla $2^{\mathrm{a}}$ interpretazione. 
Ise coordinate del polo sono rispettivamente

$$
\begin{array}{lll}
\gamma^{\prime}=74^{0}, 5 & \delta_{p}^{\prime}=7^{0}, 6 & \psi^{\prime}{ }_{p}=58^{0}, 5 \mathrm{E} \\
\gamma^{\prime \prime}=39^{0}, 2 & \delta^{\prime \prime}{ }_{p}=2^{0}, 1 & \psi^{\prime \prime}{ }_{p}=156^{\circ} \mathrm{E}
\end{array}
$$

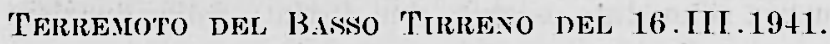

Lo studio di questo terremoto è stato eseguito da uno di noi, gli elementi calcolati sono:

$$
\begin{aligned}
& p_{o}=38^{\circ} 26^{\prime} 19^{\prime \prime} \quad \mathrm{N} \text { (latit. geografica) } \\
& \lambda_{o}=12^{\circ} 0 \overline{6}^{\prime} 23^{\prime \prime} \quad \mathrm{I} \\
& H_{o}=16^{\mathrm{\prime}} 35^{\mathrm{m}} 13^{\mathrm{s}}, 2 \\
& h^{\prime}=85 \mathrm{~km} .
\end{aligned}
$$

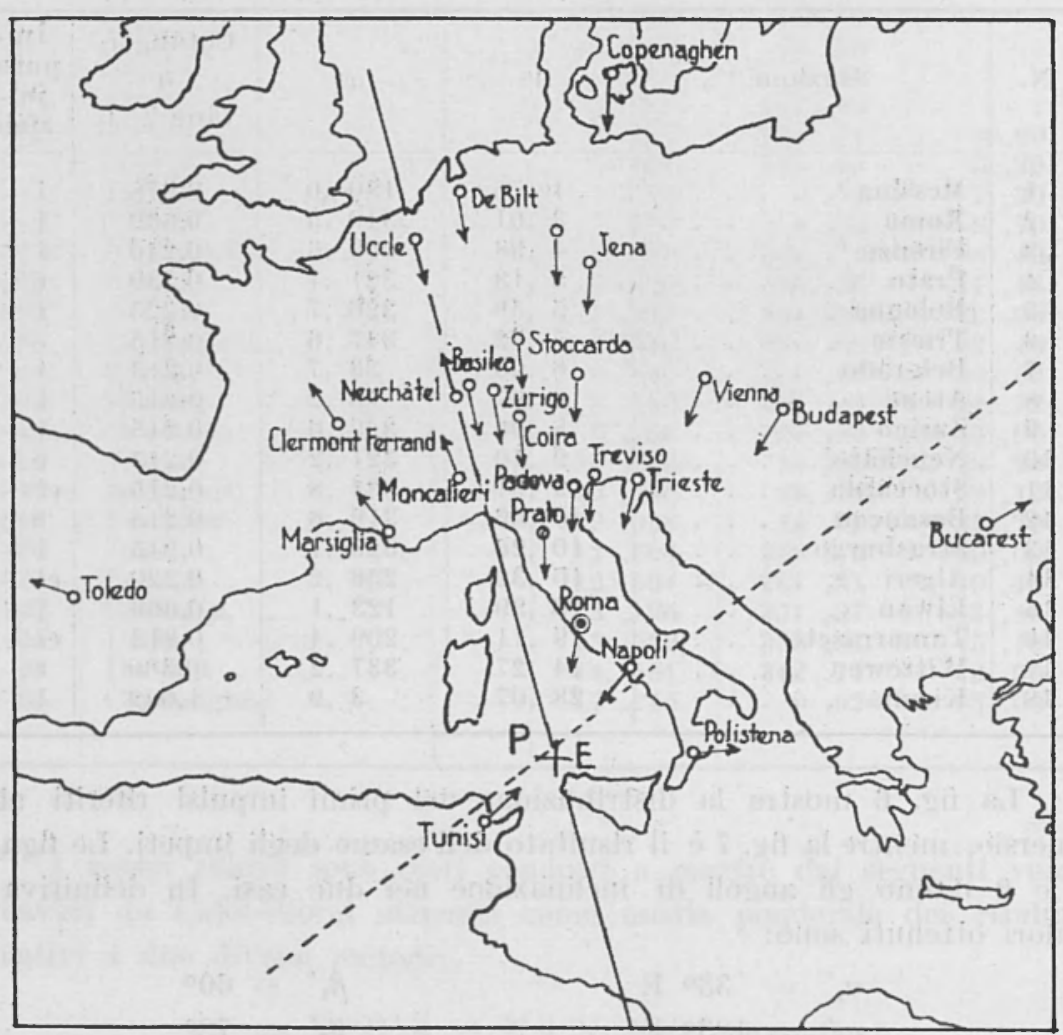

Fig. 10

Un primo esame della distribuzione degli impulsi iniziali ha condotto allo schema caratteristico della frattura, ove però la intersezione delle 
rette nodali non coincideva con l'epicentro, inoltre tali rette non risultavano perpendicolari tra loro (fig. 10).

Eseguita la proiezione stereografica sulla sfera ipocentrale in base agli elementi di cui alla tabella 2 , le proiezioni dei due cerchi risultano

Tabella 4

\begin{tabular}{|c|c|c|c|c|c|c|c|c|c|c|c|}
\hline $\mathrm{N}$. & Stazioni & & & & Ao & & & $\mu^{\circ}$ & & $\begin{array}{c}\text { Cotang } i_{h} \\
0 \\
\operatorname{tang} 1 / 21^{\prime}\end{array}$ & $\begin{array}{c}\text { Primo } \\
\text { im- } \\
\text { pulso }\end{array}$ \\
\hline I & Algreri & & . & & $5 \circ 14^{\prime}$ & $20^{\prime \prime}$ & 174 & 14 & 34 & $(0.04570)$ & + \\
\hline 2 & Pavia & . & . & & 34 & 00 & 45 & 27 & 27 & 0.19936 & - \\
\hline 3 & Bologna . & . & . & 13 & 17 & 17 & 52 & 50 & 54 & $"$ & - \\
\hline 4 & Roma . & . & . & 13 & 318 & 17 & 63 & 16 & 51 & $"$ & - \\
\hline 5 & Zurigo & . & . & 13 & 340 & 43 & 44 & 52 & 20 & $"$ & - \\
\hline 6 & Coira & . & 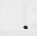 & 13 & 348 & 17 & 40 & 26 & 49 & $"$ & - \\
\hline 7 & Stoccarda. & .. & . & 14 & +26 & 51 & 35 & 07 & 52 & $"$ & - \\
\hline 8 & locle. & . & & 14 & +53 & 52 & 20 & 17 & 60 & " & - \\
\hline 9 & Messina & 8 & . & 15 & 509 & 09 & 79 & 18 & 40 & 0.29130 & - \\
\hline 10 & 'Trieste & . & 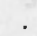 & 15 & 533 & 15 & 50 & 51 & 53 & $n$ & - \\
\hline II & De Bilt & 8 & . & 16 & 618 & 07 & 19 & 22 & 28 & 0.34400 & - \\
\hline 12 & Rathfaanham & • & • & 16 & 624 & 55 & 354 & 04 & 50 & $"$ & + \\
\hline 13 & Zagabria & 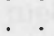 & 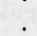 & 17 & 701 & 53 & 43 & 02 & 32 & " & $\ldots$ \\
\hline 14 & Praga. . & . & . & 18 & 22 & 07 & 39 & 03 & 27 & 0.36496 & - \\
\hline 15 & A mburgo & . & $\cdot$ & 19 & 902 & 00 & 25 & 08 & $2 i$ & 0.39829 & -.- \\
\hline 16 & Belgrado & . & & 19 & 940 & 24 & 59 & 06 & 40 & $n$ & -.. \\
\hline 17 & A tene & . & & 21 & 36 & 04 & 99 & 06 & & $"$ & - \\
\hline 18 & Tppsala & . & & 26 & 635 & 41 & 23 & 59 & 13 & 0.51835 & -- \\
\hline 19 & Reyliavik & . & & 29 & 22 & 21 & $3+3$ & 04 & 09 & 4673 & + \\
\hline 20 & Kiruna & . & & 33 & $3+1$ & 21 & 16 & 06 & 51 & 0165 & - \\
\hline 21 & Seven hills & & & 49 & 34 & 52 & 304 & 07 & 2 & 7236 & + \\
\hline 22 & Shawinigan Fall & & & 50 & 52 & 18 & 303 & +1 & 0 & 0.89988 & 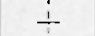 \\
\hline 23 & Weston Mass & . & & 51 & 13 & 03 & 298 & 35 & 0 & 2817 & $\div$ \\
\hline 24 & Ottawa.. & . & . & 53 & 38 & 20 & 303 & 02 & 2 & 0.95673 & - \\
\hline 2 & New York & . & . & 53 & 37 & 46 & 297 & 15 & 4 & $"$ & $\div$ \\
\hline 20 & Kirlkland. & & & 55 & 516 & 34 & 307 & 35 & 0 & 1.01465 & $\therefore$ \\
\hline 2 & Fort de France. & . & . & 57 & 702 & 50 & 283 & 02 & 0 & 1401 & + \\
\hline 28 & Merida . & 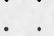 & & 74 & 42 & 32 & 284 & 48 & 4 & 1.44958 & + \\
\hline 29 & Vera ('ruz & & & 80 & 40 & 00 & 286 & 49 & 2 & 1.61388 & + \\
\hline 30 & Chihaualna & & & 81 & 155 & 43 & 299 & 56 & 2 & $"$ & + \\
\hline 31 & ast & 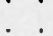 & & 84 & 432 & 35 & 311 & 58 & $\mathrm{i}$ & 1.75319 & + \\
\hline 32 & Iemaha & & & 84 & 444 & 14 & $3 \mid 3$ & 06 & & n. & \\
\hline 33 & China I ake & • & - & 85 & $5 \quad 12$ & 45 & 311 & 52 & 33 & I. 80281 & + \\
\hline 34 & Riverside & . & & 86 & 619 & 0 & 310 & 25 & 0 & " & $\dot{\varphi}$ \\
\hline 35 & Palomar & . & . & 86 & 626 & 22 & 309 & 39 & 4 & ") & + \\
\hline 36 & Berkeley & & & 86 & 628 & 31 & 315 & 58 & 0 & $"$ & + \\
\hline 37 & Ionte Wilson & . & & 86 & 634 & 10 & 310 & 57 & 4 & $"$ & + \\
\hline 38 & Pasadena. & & & 86 & $6+1$ & 56 & 310 & 50 & 0 & $"$ & + \\
\hline 39 & Taenbaia & & & 87 & 732 & 06 & 289 & 04 & 12 & " & + \\
\hline 40 & La Paz. & . & & 101 & 47 & 41 & 242 & 18 & 47 & $1.96 \mid 20$ & \\
\hline
\end{tabular}


ortogonali nell'ipocentro. Le figure 11 e 12 hanno permesso di giungere ai seguenti valori:

$$
\begin{array}{rll}
a_{1}=22^{\circ} 15^{\prime} \mathrm{W} & \beta_{1}=0^{\circ} \\
a_{2}=67^{\circ}+5^{\prime} \mathrm{E} & \beta_{2}=31^{\circ} \\
\gamma=31^{\circ} & \delta_{p}=0^{0}, 54 & \psi_{p}=157^{\circ}, 75 \mathrm{E} .
\end{array}
$$

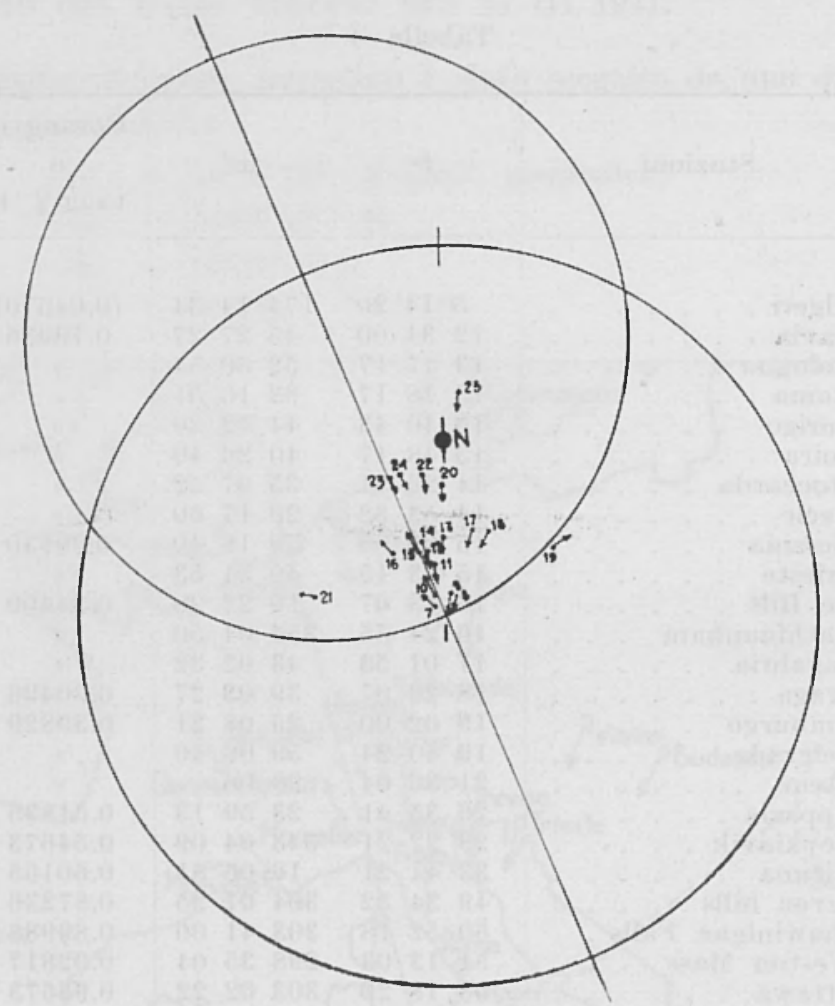

Fig. 11

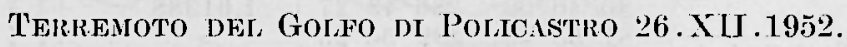

I dati ipocentrali del terremoto sono stati determinati da A. Girlanda

$$
\begin{aligned}
& \varphi_{o}^{\prime}=39^{\circ}+6^{\prime}, 279 \pm 01^{\prime}, 95 \tau \Lambda^{\top} \quad \text { (latit. geocentrica) } \\
& \varphi_{0}=399^{\circ} \tau^{\prime}, 68 \Lambda^{r} \quad \text { (latit. geografica) } \\
& \lambda_{0}=15^{\circ} 32^{\prime}, 798 \pm 04^{\prime}, 072 \mathrm{E} \\
& H_{o}=23^{\mathrm{n}} 55^{\mathrm{m}} 55^{\mathrm{s}}, 6 \pm 0^{\mathrm{s}, 6} \\
& h==264,6 \pm 10,1 \mathrm{~km} \text {. }
\end{aligned}
$$


I. valori riportati nella tabella 3 sono stati ottenuti assumendo la velocità all'epicentro pari a $8,34 \mathrm{~km} / \mathrm{sec}$ e calcolando le velocità apparenti delle $P$ per le varie distanze con le dromocrone di Jeffreys. La fig. 13 relativa alla distribuzione dei versi degli impulsi iniziali mostra che

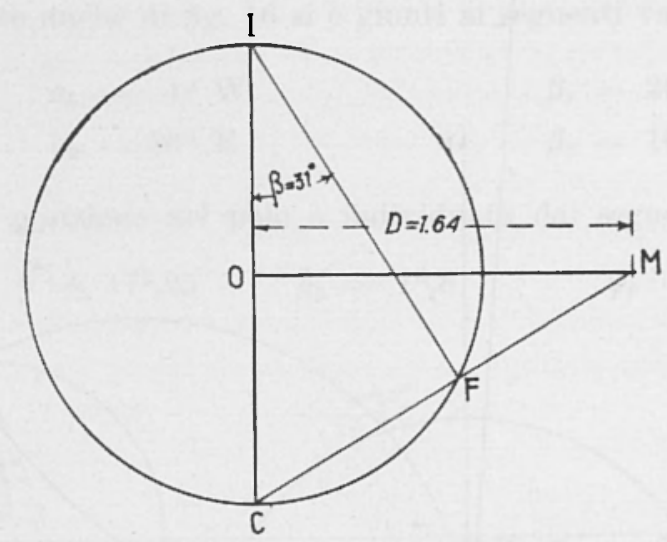

Figr. 12

anche questa volta il meccanismo che ha originato il terremoto is una frattura secondo due possibili piani i cui azimut rispetto al Nord sono

$$
a_{1}=26^{\circ} \mathrm{W}^{r}
$$

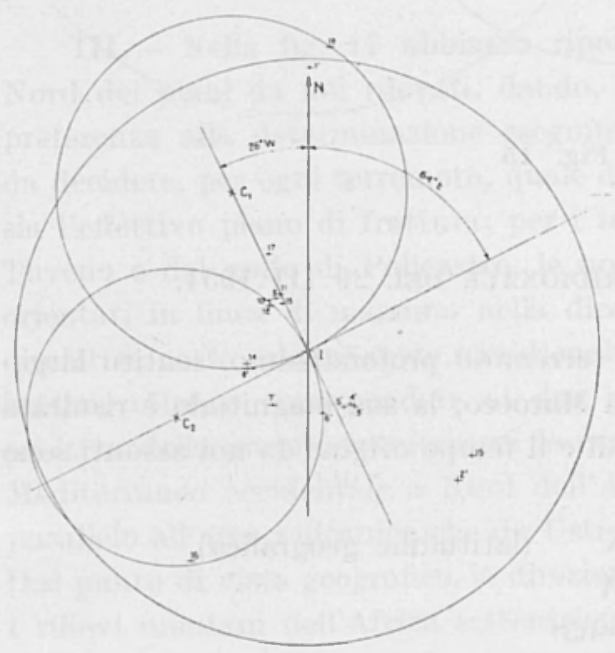

Fig. 13

$$
a_{2}=64^{\circ} \mathrm{E}
$$

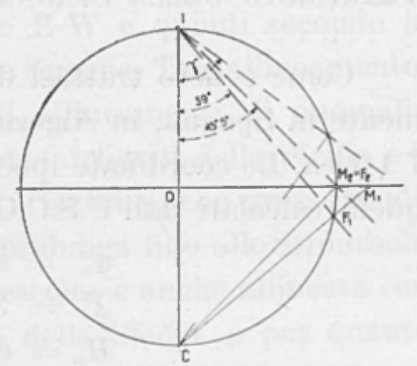

Fig. 14 
I comispondenti angoli di inclinazione e le coordinate del polo sono (fig. 1t):

$$
\beta_{1}=45,5^{\circ} ; \quad \hat{\rho}_{2}=39^{\circ} ; \quad \gamma=55^{\circ} ; \quad \delta_{p}=30,3 ; \quad \psi_{p}=284^{\circ}, \tilde{5} \mathrm{~F}
$$

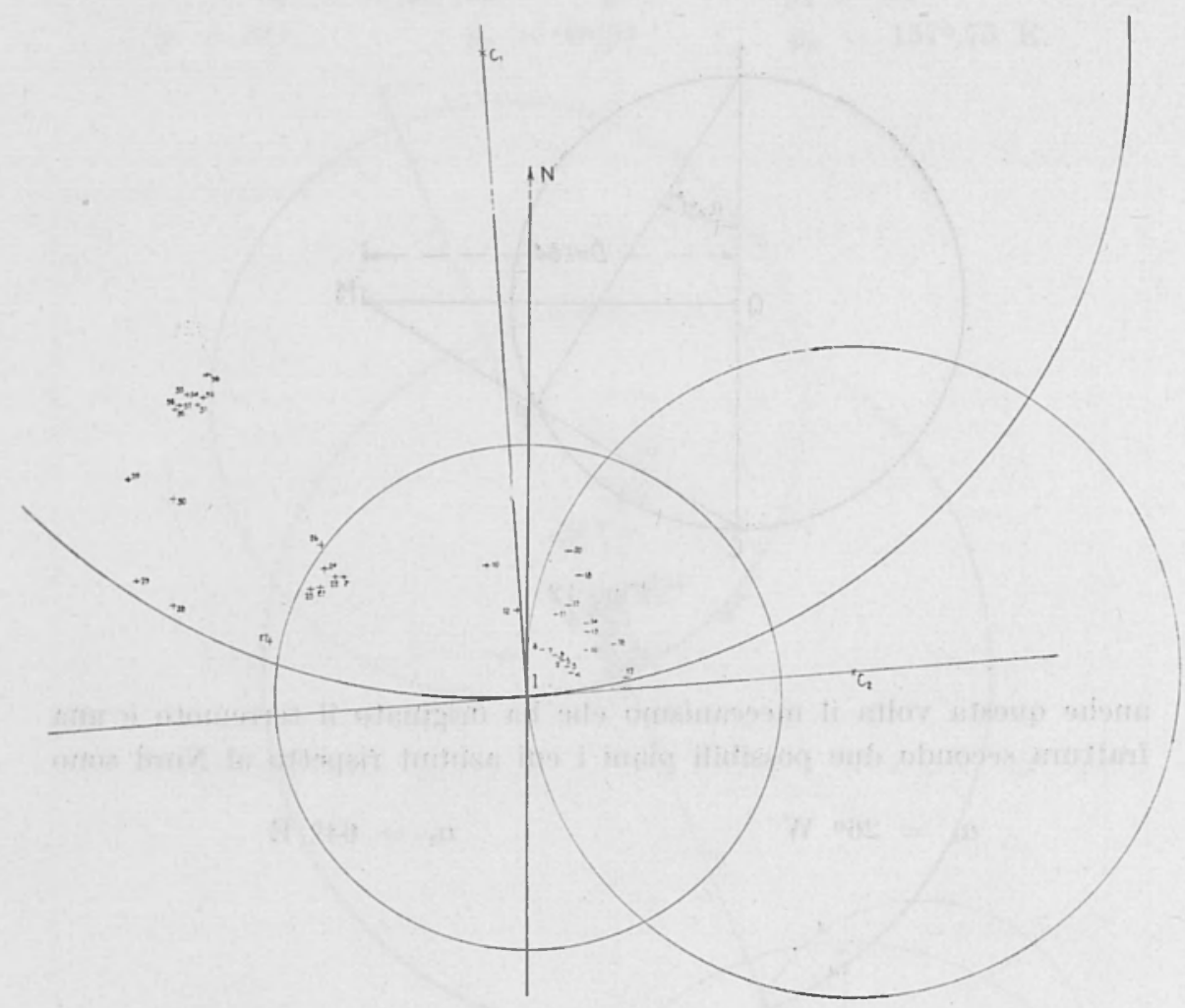

Fig. 15

Terremoto deLla Spigna meridionale DEL 29.TII.195.

Come è noto trattasi di un terremoto profondissimo, sentito largamente in Spagna, in Algeria e in Marocco; la sua magnitudo è risultata T 1/4. ca. Le coordinate ipocentrali e il tempo origine da noi assunti sono quelli calcolati dall'U.S.C.G.S.

$$
\begin{aligned}
& \varphi_{0}=37^{\circ} \mathrm{N} \quad \text { (latitudine geografica) } \\
& \lambda^{\circ}=3,5^{\circ} \mathrm{W} \\
& H_{0}=06^{{ }^{\prime} 1} 17^{\mathrm{m} 0} 05^{\mathrm{s}} \\
& \mathrm{l}=650 \mathrm{~km} .
\end{aligned}
$$


I calcoli, in coordinate geocentriche, sono stati eseguiti a partire dalla velocità $F_{h}=10,3 \mathrm{~km} / \mathrm{sec}$, che è quella calcolata da Gutenberg per $650 \mathrm{~km}$ mentre le velocità apparenti si sono ottenute interpolando le dromocrone di Jeffreys. La tabella 4 è il risultato dei calcoli stessi. Il terremoto, come mostra la fig. 15 è dovuto ad una frattura, per cui tenendo conto anche di- fig. 16 si è giunti ai seguenti valori:

$$
\begin{array}{ll}
a_{1}=4^{\circ} \mathrm{W} & \beta_{1}=20,5^{\circ} \\
a_{2}=86^{\circ} \mathrm{E} & \beta_{2}=10,5^{\circ}
\end{array}
$$

mentre la posizione del polo è individuata dai seguenti dati:

$$
\gamma=17^{\circ}, 25 \quad \delta_{p}=1^{\circ}, 8 \quad \psi_{p}=58^{\circ}, \tilde{5} \mathrm{E} .
$$

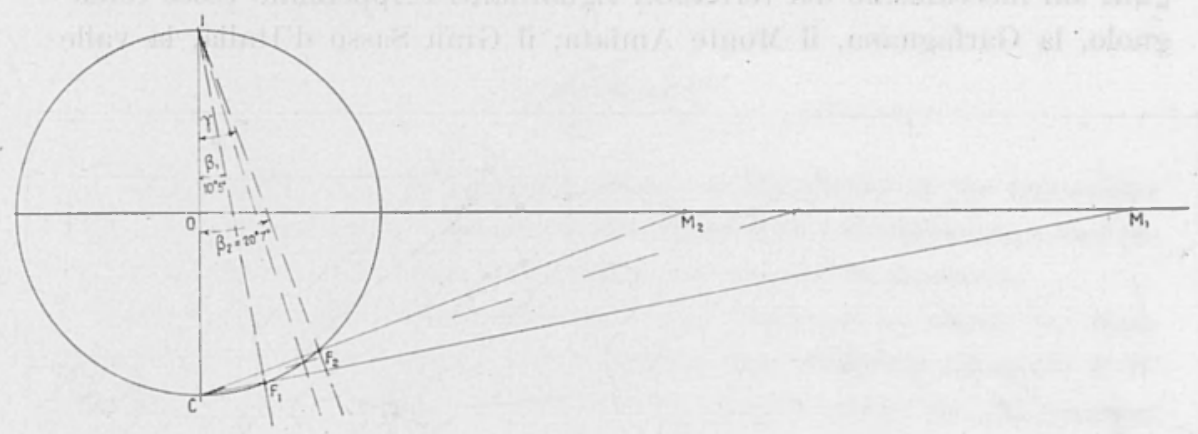

Fig. 16

III. - Nella fig. 17 abbiamo riportato l'orientamento rispetito al Ford dei piani da noi rilevati, dando, per il terremoto delle Lipari, la preferenza alla determinazione eseguita con le "emersio ". Resta ora da decidere, per ogni terremoto, quale dei due piani previsti dalla teoria, sia l'effettivo piano di frattura; per i terremoti della Spagna, del basso Tirreno e del golfo di Policastro, le nostre preferenze vanno per quelli orientati in linea di massima nella direzione E-W e quindi secondo la direttrice coste calabre-coste meridionali della Spagna. Tale allineamento, infatti, oltre a corrispondere ai due grandi allineamenti di anomalia positiva della gravità interessanti le coste settentrionali della Sicilia e il Mediterraneo oceidentale a Nord dell'Africa, risulta anche grosso modo parallelo all'arco vulcanico che da Ustica si prolunga fino allo Stromboli. T)al punto di vista geografico, la direzione prescelta è anche allineata con i rilievi montani dell'Africa settentrionale e della Sicilia, e per quanto riguarda l'Atlantico è praticamente coincidente con la direzione di uno 
dei due probabili piani di frattura riscontrati per il forte terremoto superficiale delle Azzorre del 25.XI.1941. Infatti nello studio di questo terremoto, il meccanismo della scossa all'ipocentro ha fornito per i due probabili piani di frattura le direzioni $3^{\circ} \mathrm{IV}$ e $87^{\circ} \mathrm{E}$, direzioni paticamente coincidenti con quelle calcolate per il terremoto della Spagna.

Per la parte orientale dell'allineamento, è necessario esaminare i risultati che si riferiscono al terremoto delle Iipari, e precisamente tener conto che per esso i piani di frattura probabili hanno un andamento diverso.

Questo fatto, unito all'andamento delle isoanomale della gravità, all'allineamento della zona dei vulcani attivi e all'orientamento dei rilievi montani degli Appennini e dello Stretto di Messina fa sospettare l'esistenza. di altre eventuali fratture profonde nella zona. D'altra parte gli studi eseguiti sul meccanismo dei terremoti riguardanti l'Appennino tosco-romagnolo; la Garfagnana, il Monte Amiata, il Gran Sasso d'Italia, la valle

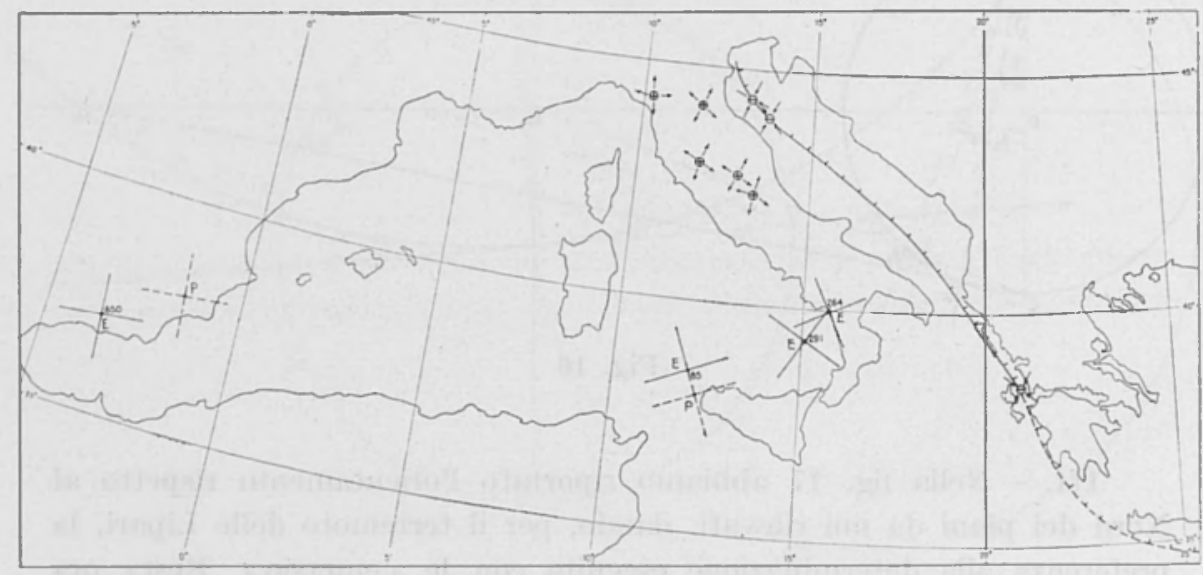

Fig. 17

dell'Aquila, l'Alto Tirreno e Cefalonia, hanno posto in evidenza che agli sprofondamenti riscontrati per le zone studiate dell'Adriatico, corrispondano dei sollevamenti della catena Appenninica.

Questo insieme di elementi pur non essendo ancora sufficiente a fornire un chiaro quadro dei fenomeni in atto, fa intravedere che per tale zona il meccanismo che origina i fenomeni sismici presenta una noterole complessità.

Roma - Ist. Naz. di Geofisica. Novembre 1959. 


\section{RIASSCNTO}

Si determina la natura fisica della scossa all'ipocentro di quattro terremoti profondi interessanti il bacino del Mediterraneo pervenendo alla conclusione che $i$ movimenti sismici sono stati originati da fratture.

Da varie considerazioni si ̀̀ preferito sceglieve per $i$ vari terremoti quel piano che risulta allineato all'incirca nella direzione EX-W (coste calabrcoste meridionali della Spagna), salvo riserve per il terremoto delle isole Lipari, dovute alla particolare posizione dell'epicentro di questo terremoto rispetto alla divezione dello stretto di Messina.

\section{ABSTRACT}

Determination of the physical nature of the shochs at the hypocentre of four deep earthquakes concerning the basin of the Mediterranean and the conclusion that these seismic movements were caused by fractures.

Owing to various considerations it was preferred to choose for these earthquakes the plane which appears aligned approximately along the $\mathbf{E}-\mathrm{W}$ direction (coasts of Calabria and Southern Spain) except for the reserves formulated in connection with the Lipari Islands due to the particular situation of the epicentre of this earthquake in respect to the direction of the Straits of Messina.

\section{BIBLIOCRAFIA}

(1) Gutenbera B., Ricitere C. F., Seismicity of the Earth.

(2) CaLoI P., Struttura geologica-sismica dell'Europa centro-meridionale ecc. "Ann. di Geofisica ", V, (1952).

(3) Caloi P., Giongi M., Studio del terremoto delle isole Lipari del 13-I T-1938. "Ann. di Geofisica ", IV. (1951).

(") Peterscinmitt S., Quelques données nouvelles sur les seismes projonds de la mer Tyrrhenienne. "Ann. di Geofisica", IX. (1956).

$\left.{ }^{5}\right)$ Di FilıpPo D., Studio microsismico del terremoto del basso Tirreno. "Boll. Soc. Sism. Ital. ", XXXIX, (1941).

$\left(^{6}\right)$ Girlanda A., Studio di un movimento sismico del basso Tirreno originante alla profondità di $265 \mathrm{~km}$. "Ann. di Geofisica ", IX, (1956). 
(7) BYqRIY P., The nature of the first motion in the Chilean earthqualie of November 11. 1922. "Am. Journal. Science" 5th ser. XVI, (1928).

$\left(^{8}\right)$ - The earthquake of July 6th 1934. Amplitudes and first motion. "Bull. Seinm. Society Amer.", 28, (1938).

$\left({ }^{\circ}\right)$ DI F'IfIPPo D., Sulla rappresentazione in superficie della natura dinamica di una scossa all'ipocentro. "Ann. di Geofisica ", III. (1950).

$\left({ }^{10}\right)$ Di FILIPPO D., Sulla rappresentazione in superficie della natura dinamica di una scossa con ipocentro profondo. "Ann. di Geofisica", III, (1950).

(11) Vecchia O., Li neamenti geofisici e geologia profonda della sicilia e delle aree circostanti. "Riv. di Geofis. Appl.", XV, (1954).

(12) 1) Filippo I)., Marcelli L., Cno studio sul terremolo di Cefalonia del 12 Agosto 1953 con particolare riguardo alla natura fisica della scossa all'ipocentro. "Ann. di Geofisica ", VII, (1954).

(13) Termier II. e Termier G., L'evolution de la lithosphère. Palis, 1957.

Direttore: Prof. Enrico Med

Responsabile: Prof. Pietro CaloI

Tipografia Pio X, Via dogli Etruschi, 7-9 - Roma - 10-2-(50 